Check for updates

Faculty of Public Health, London, UK

President@fph.org.uk

Cite this as: BMJ 2022;376:0121

http://dx.doi.org/10.1136/bmj.o121

Published: 19 January 2022

\section{Official list of covid-19 symptoms must be updated}

\section{Failure to list new variant symptoms is a threat to pandemic control in the UK}

\section{Maggie Rae president}

With the omicron variant of SARS-CoV-2 now dominant across the UK, and the World Health Organization warning that half of Europe will have been infected within the next six to eight weeks, ${ }^{1}$ we must respond and adapt our approaches to meet the challenges it poses. Although studies have found that omicron is less likely to cause severe illness than the delta variant, ${ }^{2}$ this highly infectious strain of SARS-CoV-2 has driven covid-19 rates to the highest yet seen in the UK. The NHS remains "on a war footing," 3 stretched to breaking point, with several hospitals declaring critical incidents ${ }^{4}$ because of rising pressures and staff absences.

Alongside boosting vaccination uptake, our test, trace, and isolate system remains our most important tool in tackling the spread of covid-19 and protecting the NHS. However, these measures can deliver successfully only if built on a foundation of proper messaging and public trust-and it is here that the government is continuing to fail with its lack of clear public health messaging.

That omicron infection produces milder symptoms and causes less severe illness is certainly a good thing, but this also means that it is much harder to detect, making an effective testing programme more crucial than ever. Given that we now know from research such as the ZOE study that the primary symptoms of the omicron variant include runny nose, headache, fatigue, sneezing, and sore throat, ${ }^{5}$ it is profoundly unhelpful that the government has not updated its official-and now outdated-list of symptoms, which highlights high temperature, a new continuous cough, or a loss or change to sense of smell or taste.

Across the world, government agencies, including WHO and the US Centers for Disease Control and Prevention, have updated their case definition of covid-19 to include more diverse symptoms, thus greatly reducing the threshold for people to test and isolate when appropriate. Unless the UK follows the clear evidence on the symptoms caused by the omicron variant and updates its guidance in step with global partners, we will see the effectiveness of our response programme compromised by an unfit case definition.

\section{Keeping up with the virus}

Similar to improving domestic and international vaccine uptake, the importance of a well functioning testing regime cannot be overemphasised in our efforts to tackle covid-19. The Scientific Advisory Group for Emergencies recommends that we prioritise the rapid testing of symptomatic people over testing asymptomatic people in outbreak areas to have the greatest effect on identifying cases and reducing transmission. ${ }^{6}$ With the government's definition of covid-19 symptoms not reflecting the evidence on omicron, infected people are not testing or isolating and are continuing to spread this highly infectious virus.

Of course, if government listens to evidence and expands the case definition for covid-19, the number of people testing and isolating will increase. This likely increase in demand must be matched with increased capacity within the testing system. Providers have warned that pharmacies across the UK have faced shortages of lateral flow tests in recent weeks, ${ }^{7}$ and in the run-up to the Christmas holidays people in England were often unable to order home test kits, an unacceptable and dangerous situation. To support and help the public in taking every action possible to prevent the spread of covid-19, the government must urgently remedy these procurement and distribution problems.

As well as a well functioning testing system, there must be better support for those required to isolate as a result of a positive test result. Throughout the pandemic we have seen the clear consequences of health inequalities play out, with the communities most at risk of serious illness from covid-19 also the least likely to come forward for testing and vaccination. ${ }^{8}$ Although some financial support is available for those who need it to self- isolate, these packages do not go far enough, or move fast enough, to offer the safety net needed by individuals and families in our most disadvantaged communities.

Our mission to control and prevent the spread of covid-19 to save lives and protect the NHS remains unchanged, and boosting vaccine uptake alongside our test, trace, and isolate system remain our most important tools in achieving this aim. But if these systems are to continue to deliver results, they must be supported by timely and accurate public health messaging. Not following clear evidence and updating the case definition for covid-19 is a failure of government that threatens the effectiveness of the measures we have put in place to control the spread of the disease.

Competing interests: We have read and understood BMJ policy on declaration of interests and have no relevant interests to declare.

Provenance and peer review: Commissioned; not externally peer reviewed.

World Health Organization. Covid-19 virtual press briefing 11 January 2022 https://twitter.com/i/broadcasts/1rmGPgElrnmKN

2 Christie B.Covid-19: Early studies give hope omicron is milder than other variants. BMJ 2021;375:n3144. doi: 10.1136/bmj.n3144 pmid: 34949600

NHS England. NHS plans new Nightingale facilities in response to Omicron. 2021 https://www.england.nhs.uk/2021/12/nhs-plans-new-nightingale-facilities-in-response-to-omicron/ 
4 Torjesen I. Covid-19: Many hospitals "are not declaring critical incidents" despite severe pressures. BM/2022;376:060. doi: 10.1136/bmj.060 pmid: 35017222

5 ZOE Study. What are the new top 5 covid symptoms? 23 Jun 2021. https://covid.joinzoe.com/post/new-top-5-covid-symptoms\#part_1

6 SAGE. 56th meeting on COVID-19.10 Sep 2020. https://assets.publishing.service.gov.uk/government/uploads/system/uploads/attachment_data/file/928699/S0740_Fifty-sixth_SAGE_meeting_on_Covid-19.pdf

7 Clews G. Pharmacies experiencing 'patchy' supplies of lateral flow tests as demand soars. Pharm J2021;307. doi: 10.1211/PJ.2021.1.121837

8 UK Government. Final report on progress to address covid-19 health inequalities. 2021.

https://www.gov.uk/government/publications/final-report-on-progress-to-address-covid-19-

health-inequalities/final-report-on-progress-to-address-covid-19-health-inequalities 